\title{
Why Noise and Dispersion may Seriously Hamper Nonlinear Frequency-Division Multiplexing
}

\author{
Stella Civelli, Enrico Forestieri, Member, IEEE, and Marco Secondini, Member, IEEE
}

\begin{abstract}
The performance of optical fiber systems based on nonlinear frequency-division multiplexing (NFDM) or on more conventional transmission techniques is compared through numerical simulations. Some critical issues affecting NFDM systems-namely, the strict requirements needed to avoid burst interaction due to signal dispersion and the unfavorable dependence of performance on burst length-are investigated, highlighting their potentially disruptive effect in terms of spectral efficiency. Two digital processing techniques are finally proposed to halve the guard time between NFDM symbol bursts and reduce the size of the processing window at the receiver, increasing spectral efficiency and reducing computational complexity.
\end{abstract}

Index Terms-Optical fiber communication, nonlinear Fourier transform, nonlinear frequency division multiplexing.

\section{INTRODUCTION}

$\mathbf{N}$ ONLINEAR frequency-division multiplexing (NFDM) has recently attracted attention as a way to cope with nonlinear effects in optical fiber communications [1]-[4]. By using the nonlinear Fourier transform (NFT) the optical signal can be represented through its nonlinear spectrum, whose evolution along the fiber is governed by a simple linear equation [1]. This is exploited in NFDM systems by encoding information directly onto the nonlinear spectrum, such that it can be easily retrieved at the receiver avoiding nonlinear interference due to propagation.

Different flavors of NFDM do exist, depending on the considered NFT boundary conditions and on the way information is mapped onto the nonlinear spectrum [4]. So far, vanishing boundary conditions have been mostly used [1], [2], the only exception (to the best of our knowledge) being the periodic boundary conditions employed in [5]. With vanishing boundary conditions, the nonlinear spectrum has a continuous part, analogous to the linear spectrum, and some discrete components (solitons) arising for specific input profiles and with no linear counterpart. Accordingly, different NFDM schemes have been proposed in which information is encoded on the continuous spectrum [2], discrete spectrum [1], or both [6].

Despite many recent theoretical and experimental publications on the subject, a clear indication of whether NFDM can actually outperform conventional systems is still missing in the literature. Moreover, we believe that the impact of some potentially critical issues has been overlooked. Thus, we

S. Civelli, E. Forestieri, and M. Secondini are with the Tecip Institute, Scuola Superiore Sant'Anna, Pisa, Italy and with the National Laboratory of Photonic Networks, CNIT, Pisa, Italy (e-mail: stella.civelli@santannapisa.it).

Part of this work was presented at the Munich Workshop on Information Theory of Optical Fiber (MIO) 2016 and at the Progress In Electromagnetics Research Symposium (PIERS) 2017, Saint Petersburg, Russia.

This work was supported in part by Fondazione Cassa di Risparmio di Firenze, under the grant NOSTRUM: NOnlinear SpecTRUm Modulation. compare here the performance of NFDM systems with that of conventional ones employing ideal electronic dispersion compensation (EDC) or digital backpropagation (DBP), focusing on the strict limitations imposed by temporal broadening (due to fiber dispersion) and nonlinear spectrum perturbation (due to amplifier noise) to NFDM systems. We consider vanishing boundary conditions and modulation of the continuous spectrum, following the nonlinear inverse synthesis (NIS) approach [2], [3]. The first choice is due to the higher simplicity and maturity of the underlying theory; the second one is due to our belief that the modulation of the continuous spectrum is essential to achieve high spectral efficiencies 1

Nevertheless, as we will show in the following, also the NIS technique suffers from some important drawbacks that, if not clearly understood and overcome, may severely limit performance and spectral efficiency. In fact, transmission is organized in bursts of $N_{b}$ information symbols, separated by a guard time $\left(N_{z}\right.$ spaces) to avoid burst interaction during propagation. The guard time: $i$ ) plays a role similar to the cyclic prefix in OFDM (emulating, at least within the limit of channel memory, the boundary conditions required by the underlying theory); ii) should at least equal the maximum time broadening induced by fiber dispersion; and iii) causes a reduction of the overall spectral efficiency by a factor $\eta=N_{b} /\left(N_{z}+N_{b}\right)$-in the following referred to as rate efficiency. We show by numerical simulations that the simple solution (used in conventional OFDM) of increasing $N_{b}$ to limit the loss of spectral efficiency is not feasible, because the performance decreases with $N_{b}$ due to a sort of signalnoise interaction taking place at the receiver. This behavior is in agreement with a recent analytical model [7], and is substantially different from the behavior of conventional systems, whose performance saturates to a finite value when $N_{b} \rightarrow \infty(\eta \rightarrow 1)$. Moreover, the computational complexity of the NFT remains a major concern for the implementation of NFDM systems, despite some recent progresses toward its reduction (see [4] and references therein). With this in mind, we investigate two techniques aimed at mitigating the impact of time broadening on spectral efficiency and computational complexity. We show, through numerical simulations, that a precompensation technique can halve the necessary guard time between different bursts, and that a windowing technique can significantly shorten the length of the processed signal without performance degradation or even provide a small gain.

\footnotetext{
${ }^{1}$ The NIS technique is a nonlinear analogous of orthogonal frequencydivision multiplexing (OFDM), tending to it in the linear regime [2]. Thus, it can be combined with conventional coding and modulation to achieve high spectral efficiencies and approach channel capacity in the linear regime.
} 


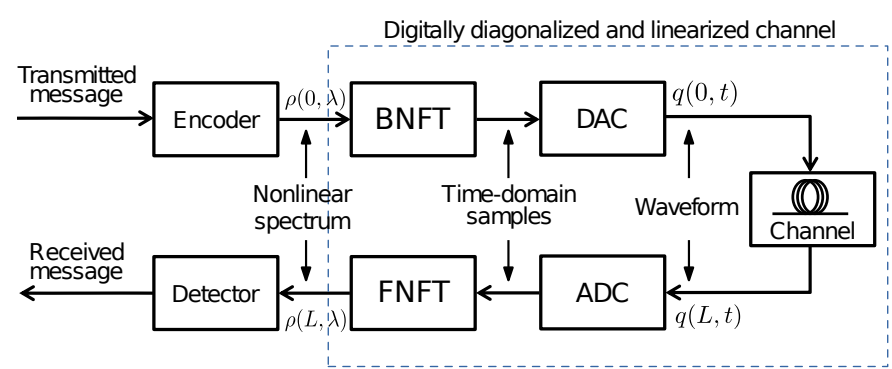

Fig. 1. NFDM transmission scheme

\section{SYSTEM PERFORMANCE}

The considered NFDM transmission scheme is sketched in Fig.1 and is based on the NIS technique proposed in [2]. The transmitter (TX) encodes the information on a quadrature phase-shift keying (QPSK) signal, whose Fourier transform (FT) is mapped onto the continuous part of the input nonlinear spectrum $\rho(0, \lambda)$. The backward NFT (BNFT) block generates, through a digital-to-analog converter (DAC), the corresponding optical signal $q(0, t)$. While $q(z, t)$ evolves along the fiber according to the nonlinear Schrödinger equation (NLSE), its nonlinear spectrum evolves linearly according to $\rho(z, \lambda)=\rho(0, \lambda) e^{-j 4 \lambda^{2} z}$ [1]. At the receiver (RX), the output optical signal $q(L, t)$ is sampled by the analog-to-digital converter (ADC) and sent to the forward NFT (FNFT) block, which computes the corresponding output nonlinear spectrum $\rho(L, \lambda)$ (corrupted by amplifier noise during propagation). Finally, the detector multiplies $\rho(L, \lambda)$ by $e^{j 4 \lambda^{2} L}$ to remove the propagation effect; performs an inverse FT, followed by matched filtering and symbol-time sampling to obtain a noisy replica of the transmitted symbols; and makes decisions based on a minimum Euclidean distance criterion 2

The QPSK signal power spectral density is raised-cosine shaped with roll-off factor $\beta=0.2$ (a typical choice in conventional systems), while the symbol rate is $R_{s}=1 / T_{s}=$ $50 \mathrm{GBd}$. The physical channel is a standard single-mode fiber of length $L=2000 \mathrm{~km}$, attenuation $\alpha=0.2 \mathrm{~dB} / \mathrm{km}$, dispersion $\beta_{2}=-20.39 \mathrm{ps}^{2} / \mathrm{km}$, and nonlinear coefficient $\gamma=1.22 \mathrm{~W}^{-1} \mathrm{~km}^{-1}$, along which ideal distributed amplification with spontaneous emission factor $\eta_{\mathrm{sp}}=4$ is considered. The bandwidth of both the DAC and the ADC is $100 \mathrm{GHz}$. The BNFT is computed by an enhanced version of the Nyström method [8], while the Layer-Peeling (LP) method is employed for the FNFT [1], [2]. An oversampling factor of 4 samples per symbol was considered for both BNFT and FNFT, unless otherwise specified. At high powers, some rare but disruptive numerical instabilities in the calculation of the noisy nonlinear spectrum were observed [9]. We conjecture they are related to the rise of discrete components in the nonlinear spectrum (solitons) induced by noise [10]. This issue has been practically resolved by resorting to linear interpolation between adjacent frequencies when the phenomenon occurs at a given frequency. As explained later, this numerical expedient is not

\footnotetext{
${ }^{2}$ Besides its simplicity and widespread use, this detection strategy achieves the capacity bound in [7] and is asymptotically optimal at low power. However, a better performance might be achievable by a more accurate detection strategy, accounting for the actual statistics of the received nonlinear spectrum.
}

(a)
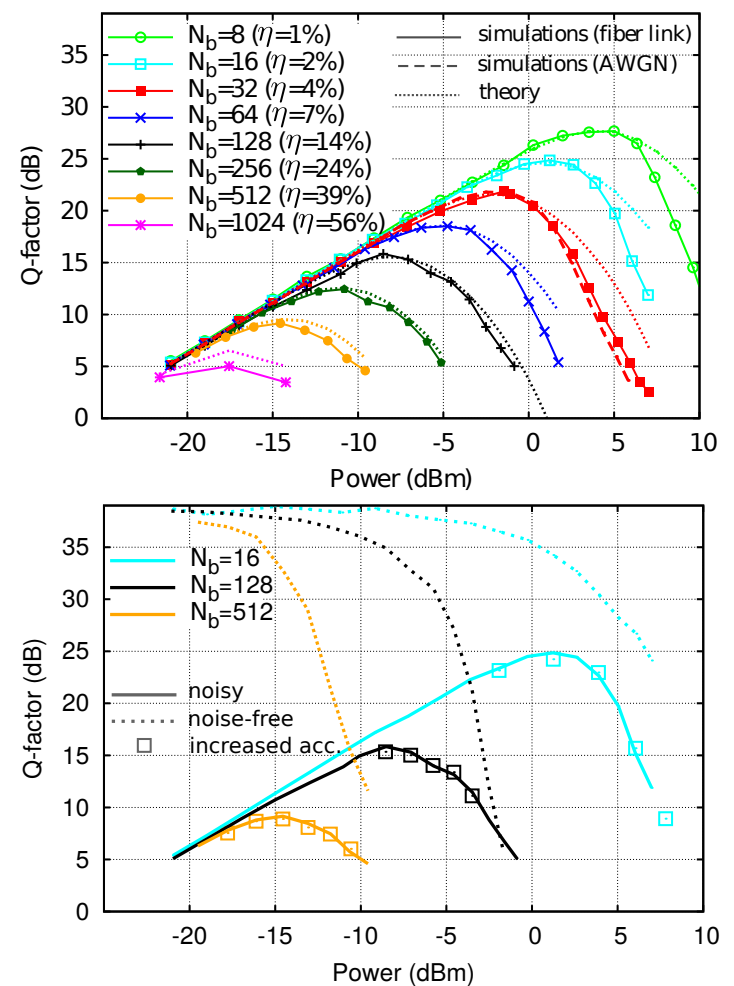

Fig. 2. (a) Q-factor vs optical power for standard NFDM with different burst length $N_{b}$ (and rate efficiency $\eta$ ); (b) Impact of numerical inaccuracies on the Q-factor.

required when employing the windowing technique proposed in the next section.

Fig. 22a) shows the Q-factor from numerical simulations (solid lines) or from the effective signal-to-noise ratio (SNR) of the analytical model in [7] (dotted lines), for different burst lengths $N_{b}$ and a guard interval of $N_{z}=800$ zeros. As required, this value is slightly larger than the overall channel memory set by the time broadening induced by dispersion, which is of the order of $2 \pi\left|\beta_{2}\right| L R_{s}^{2}(1+\beta)$ symbol times $(\sim 768 \text { in our case })^{3}$. The corresponding rate efficiencies $\eta$ are also indicated. After reaching a maximum at some optimum power, all curves fall down since the impact of amplifier noise on the nonlinear spectrum increases with signal energy (a sort of signal-noise interaction). The impact of numerical inaccuracies, already considered in [9], is shown in Fig.2]b), where the simulation results of Fig.2 a) are compared with the results obtained in the corresponding noise-free scenario and with those obtained with higher sampling rate ( 16 samples per symbol) and longer guard time $\left(N_{z}=900\right.$ guard symbols). It is apparent that, in the region near the optimal power, the noise-free curves are above the noisy ones; moreover, the performance remains unchanged if a higher accuracy is considered. Therefore, Fig.2 (b) confirms that the observed performance degradation is due to the interaction of signal and noise, rather than to numerical inaccuracies. The agreement between theory and simulations up to the optimum power further confirms that the obtained results are not affected by

\footnotetext{
${ }^{3}$ The initial broadening induced by the BNFT at the receiver is usually negligible compared to that induced by dispersion, as shown in [9]. This is not true for the lowest curve of Fig. 22 a) $\left(N_{b}=1024\right)$, such that an additional penalty is observed compared to the analytical model.
} 
(a)

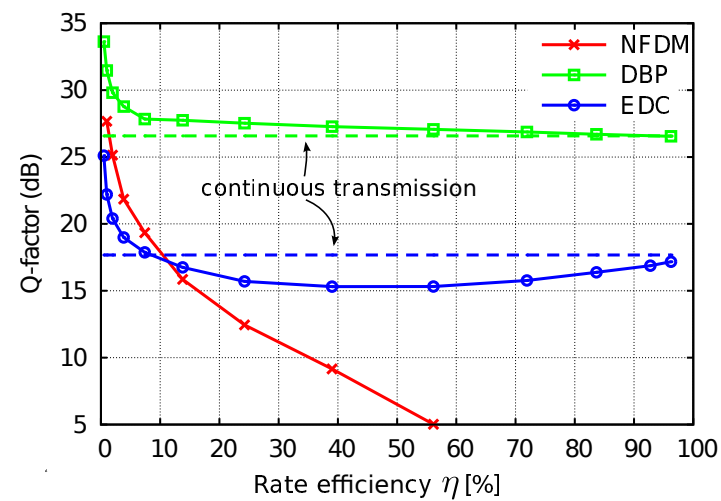

(b)

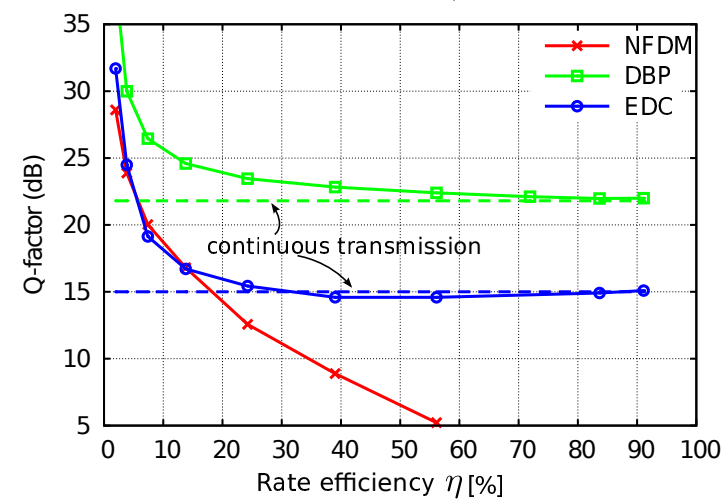

Fig. 3. (a) optimal Q-factor vs rate efficiency for NFDM and conventional systems; (b) optimal Q-factor vs rate efficiency for NFDM and conventional systems with $\beta_{2}=-1.27 \mathrm{ps}^{2} / \mathrm{km}$.

limitations of the numerical algorithms. Moreover, it verifies the accuracy of the perturbation approach and asymptotic approximations used in [7] for the computation of the effective SNR. Remarkably, the maximum reduces as $N_{b}$ increases. This behavior persists for bursts longer than the channel memory, as shown for $N_{b}=1024$. Interestingly, unlike conventional systems, the performance of the NFDM scheme considered here remains unchanged (at least in the considered range of powers) if the optical fiber channel is replaced with an additive white gaussian noise (AWGN) channel with same accumulated noise (shown with dashed line for $N_{b}=32$; results are similar for any $N_{b}$ ). We will return on this later.

To better understand this issue and its relevance, the maximum of each curve in Fig.2 a) is reported in Fig. 3. a) as a function of the rate efficiency $\eta$, along with the corresponding performance of ideal DBP and EDC methods. For the sake of comparison, burst mode transmission with $N_{z}=800$ and same modulation parameters were considered in all cases. As expected, the performance of both DBP and EDC converges to that of a continuous transmission for bursts longer than the channel memory $\left(N_{b}>N_{z}\right.$, corresponding to $\left.\eta>0.5\right)$. This is because, in these systems, nonlinear interaction involves only signal and noise components that are closer in time than the overall channel memory. On the other hand, the NFDM performance keeps decreasing even for longer bursts, as in this case signal-noise interaction does occur during fiber propagation, as in conventional systems, but at the receiver over the full integration window used for computing the nonlinear spectrum (the FNFT), with an impact that increases with the total signal energy therein. This is confirmed by the

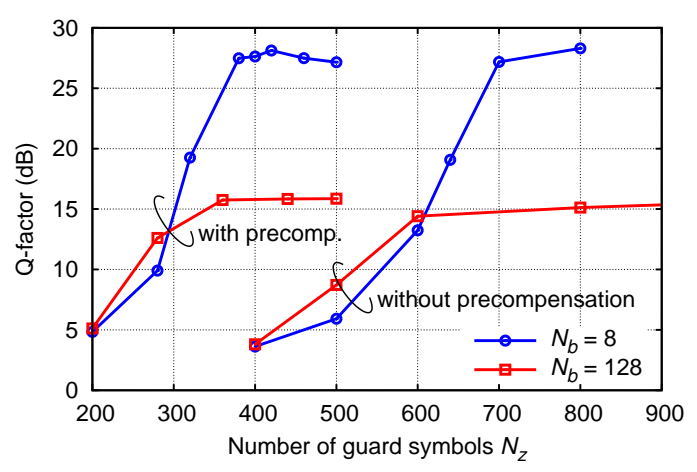

Fig. 4. Optimal Q-factor vs guard interval with and without precompensation.

curve shown in Fig.22(a) for the NFDM system over an AWGN channel. The comparison in Fig.3 a) also reveals that ideal DBP performs better than NFDM, which performs better than EDC only for bursts of short-medium length.

In the single-user scenario considered here, its worse performance with respect to ideal DBP may be not a crucial issue, as NFDM is expected to perform better in multiuser scenarios, where DBP is much less effective because of inter-channel interference [11]. A more critical issue is the peculiar dependence of its performance on burst length, as also confirmed by theory. In fact, as shown in Fig.3. a), a reasonable performance is obtained only at the expense of a low rate efficiency. For $\eta>0.11$, NFDM performs worse than simple EDC, definitely loosing any appeal. Moreover, also the computational complexity of most practical NFT algorithms has an unfavorable dependence on the total signal length (burst length plus guard time). Note that, if there were no broadening, a much shorter guard time and burst length could be considered, with a significant improvement in performance and complexity. Thus, one is tempted to check whether it is any better in links with low-dispersion fibers. Considering a sixteen times lower dispersion $\left(\beta_{2}=-1.27 \mathrm{ps}^{2} / \mathrm{km}\right)$ and guard time $\left(N_{z}=50\right)$, Fig. 3 b b shows that the dependence of NFDM performance on the rate efficiency remains practically unchanged (signal-noise interaction in the FNFT is reduced for a shorter burst, but is increased for a lower dispersion, the two effects canceling out). Instead, DBP and EDC performance worsens at high rates and slightly improves at low rates. The overall picture does not change significantly: DBP outperforms NFDM and EDC, whose performance is almost the same up to $\eta=0.14$. For $\eta>0.14$, NFDM performance degrades much faster than EDC and DBP, as in the previous scenario.

\section{PRecompensation AND WINDOWInG}

The large number of guard spaces $N_{z}$ required to avoid burst interaction during propagation reduces the transmission rate by the factor $\eta=N_{b} /\left(N_{z}+N_{b}\right)$, with a significant loss of spectral efficiency. For mitigating this loss, we introduce a precompensation technique that, by minimizing the time broadening induced by dispersion throughout the link, allows to reduce $N_{z} 4$ In order to remove the propagation effect from the received nonlinear spectrum $\rho(L, \lambda)$, rather than

\footnotetext{
${ }^{4}$ The same technique has been independently proposed in [12].
} 
multiplying it by $e^{j 4 \lambda^{2} L}$ as done in standard NIS, we split the compensation between TX and RX, both pre-multiplying $\rho(0, \lambda)$ and post-multiplying $\rho(L, \lambda)$ by $e^{j 2 \lambda^{2} L}$. This is the same as generating the signal at a distance $-L / 2$ and propagating it to a distance $L / 2$. In this way, the same time broadening of $\pi\left|\beta_{2}\right| L R_{s}^{2}(1+\beta)$ symbol times is observed at TX and RX, in fact halved with respect to the standard implementation.

Fig. 4 shows the Q-factor obtained with and without precompensation for bursts of length $N_{b}=8$ and $N_{b}=128$ at their optimal launch power (about $3.8 \mathrm{dBm}$ and $-8.5 \mathrm{dBm}$, respectively) as a function of the number of guard symbols $N_{z}$. For both burst lengths, precompensation allows using half the guard time to achieve the same performance, with a significant increase of the rate efficiency (almost doubled). This precompensation technique reduces the computational complexity of the FNFT while increasing the BNFT one (as the total processing windows are, respectively, shortened and lengthened), with an overall effect that depends on the algorithms employed for the BNFT and FNFT.

As mentioned above, time broadening affects also the computational complexity of the FNFT at RX, which, in principle, must be performed for each burst on the entire time range $-T / 2<t<T / 2$, with $T=\left(N_{b}+N_{z}\right) T_{s}$. Nevertheless, some computational savings can be achieved by noting that, similarly to the linear spectrum, also the continuous part of the nonlinear spectrum experiences a sort of group velocity dispersion during propagation, with different frequency components traveling at different speeds. As a result, different time portions of the received optical signal bring information about different spectral components of the nonlinear spectrum. This is illustrated in Fig.5 5 a), which shows the modulus of the nonlinear spectrum (vertical axis) as obtained when applying the LP algorithm to the received optical signal truncated to the time interval $-T / 2<t<\tau$, with the upper limit reported on the $\tau$ axis. Three different spectral components $\lambda$ are reported at different depths in the graph. For illustration purposes, results are shown in the absence of optical noise. It is apparent that, for each spectral component $\lambda$, only a small portion of the received optical signal - contained in a time window whose center depends linearly on the considered frequency - contributes to the final value of the nonlinear spectrum. This suggests the following windowing technique: given the received optical signal, each frequency component $\rho(L, \lambda)$ is computed by applying the LP method on the moving time window $\max \left\{t_{\lambda}-T_{w} / 2,-T / 2\right\}<t<\min \left\{t_{\lambda}+T_{w} / 2, T / 2\right\}$, where $T_{w}<T$ is the window width (to be optimized) and $t_{\lambda}=-2 \beta_{2} L \lambda / T_{0}$ its center, $T_{0}$ being the time normalization parameter used to define the NFT [1].

Fig. 5 b) shows the Q-factor at optimum power obtained by the described windowing technique as a function of the window width $T_{w}$, for the same system in Fig. 2 (a). These results show that the time window for computing the FNFT can be reduced to about $70 \%, 50 \%, 20 \%$, and $40 \%$ of the total signal duration for $N_{b}=8,32,128$, and 1024 , respectively, with significant computational savings. The different behavior for different burst lengths depends on the maximum achievable Q-factor and on the initial burst length: the lower the Q-factor, the narrower the time window where the signal contribution
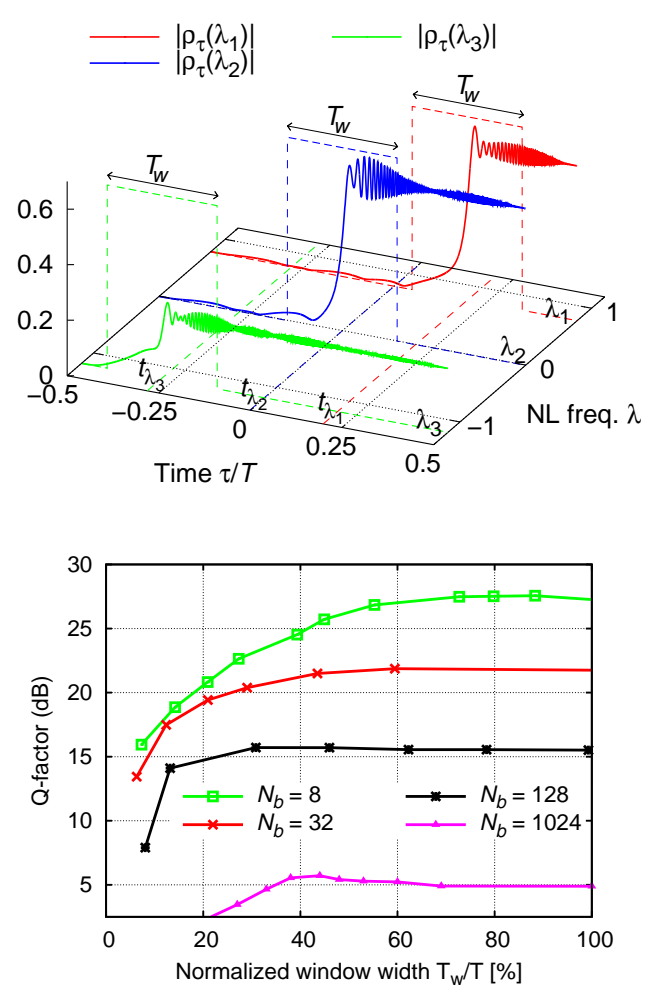

Fig. 5. (a) Modulus of the noiseless nonlinear spectrum (vertical axis) vs upper time limit considered in the LP algorithm ( $\tau$ axis) for different spectral components; (b) optimal Q-factor when using the windowing technique vs window width.

dominates over noise, until the window width becomes much smaller than $T_{s} N_{b}$.

Moreover, this technique avoids the excess noise outside the window of interest for each considered frequency, slightly improving performance (as shown for $N_{b}=1024$ in Fig. 5 b b)) and reducing the numerical instabilities of the LP algorithm mentioned in the previous section. In fact, when using the windowing technique, we were able to reproduce the same results of Fig. 2(a) without the need to resort to the interpolation expedient and almost avoiding the small penalty (compared to the theoretical curves) observed in Fig. 22(a) for $N_{b}=1024$ near the optimum launch power.

\section{CONCLUSIONS}

Some critical issues arising in NFDM systems based on vanishing boundary conditions and modulation of the continuous spectrum have been investigated. According to current theory, these systems operate in burst mode, with a guard time between bursts long enough to accommodate the temporal broadening induced by the accumulated dispersion. Our findings show that, unlike in conventional systems, in the NIS scheme considered in this work, the spectral efficiency loss due to the guard time cannot be reduced by increasing at will the burst length. In fact, the performance degrades as the burst length increases due to a detrimental signal-noise interaction taking place in the FNFT operation at the receiver. The impact of this effect is such that, in a 50 GBd QPSK NIS system over $2000 \mathrm{~km}$ of standard fiber, the effective rate cannot be increased to more than $11 \mathrm{~Gb} / \mathrm{s}$ (about 100 information symbols 
and 800 guard symbols) without degrading its performance below that of a conventional system with simple EDC. Similar results are found when considering low-dispersion fibers $(D=$ $1 \mathrm{ps} / \mathrm{nm} / \mathrm{km}$ ). A digital precompensation technique has been proposed to halve the guard time required between different bursts of information symbols, allowing to almost double the NFDM spectral efficiency. Moreover, a windowing technique has been introduced to limit the computational complexity at the RX and to avoid excess noise entering the FNFT computation in the presence of temporal broadening. These improvements, though significant, are still not sufficient to make NIS an attractive replacement to conventional systems. In order to pave the way for the advent of NFDM systems and exploit their great potentials [11], the critical issue highlighted in this work should be solved, for instance by devising more appropriate detection strategies based on the actual statistics of the noisy nonlinear spectrum.

\section{REFERENCES}

[1] M. I. Yousefi and F. R. Kschischang, "Information transmission using the nonlinear Fourier transform, Parts I-III," IEEE Trans. Inf. Theory, vol. 60, pp. 4312-4369, July 2014.

[2] S. T. Le, J. E. Prilepsky, and S. K. Turitsyn, "Nonlinear inverse synthesis for high spectral efficiency transmission in optical fibers," Opt. Express, vol. 22, no. 22, pp. 26720-26741, 2014.

[3] S. T. Le, I. D. Philips, J. E. Prilepsky, P. Harper, A. D. Ellis, and S. K. Turitsyn, "Demonstration of nonlinear inverse synthesis transmission over transoceanic distances," J. Lightwave Technol., vol. 34, no. 10, pp. 2459-2466, 2016.

[4] S. K. Turitsyn, J. E. Prilepsky, S. T. Le, S. Wahls, L. L. Frumin, M. Kamalian, and S. A. Derevyanko, "Nonlinear fourier transform for optical data processing and transmission: advances and perspectives," Optica, vol. 4, no. 3, pp. 307-322, 2017.

[5] M. Kamalian, J. E. Prilepsky, S. T. Le, and S. K. Turitsyn, "Periodic nonlinear fourier transform for fiber-optic ccommunication, part ii: Eigenvalue communication," Opt. Express, vol. 24, no. 16, pp. 18370$18381,2016$.

[6] V. Aref, S. T. Le, and H. Buelow, "Demonstration of fully nonlinear spectrum modulated system in the highly nonlinear optical transmission regime," European Conference on Optical Communication (ECOC 2016), Proceedings of, 2016.

[7] S. A. Derevyanko, J. E. Prilepsky, and S. K. Turitsyn, "Capacity estimates for optical transmission based on the nonlinear fourier transform," Nature Communications, 2016.

[8] S. Civelli, L. Barletti, and M. Secondini, "Numerical methods for the inverse nonlinear Fourier transform," in Proc. Tyrrhenian Int. Workshop Digital Communications (TIWDC), (Florence, Italy), pp. 13-16, Sept. 2015.

[9] S. Civelli, E. Forestieri, and M. Secondini, "Impact of discretizations and boundary conditions in nonlinear frequency-domain multiplexing," in 18th Italian National Conference on Photonic Technologies (Fotonica 2016), 2016

[10] H. Bülow, "Experimental demonstration of optical signal detection using nonlinear fourier transform," Journal of Lightwave Technology, vol. 33, no. 7, pp. 1433-1439, 2015.

[11] M. I. Yousefi and X. Yangzhang, "Linear and nonlinear frequencydivision multiplexing," European Conference on Optical Communication (ECOC 2016), Proceedings of, 2016.

[12] I. Tavakkolnia and M. Safari, "Dispersion pre-compensation for nftbased optical fiber communication systems," in Conference on Lasers and Electro-Optics, OSA Technical Digest, 2016. 